\title{
Effect of fluid resuscitation with balanced solutions on platelets: In vitro simulation of $20 \%$ volume substitution
}

\author{
Łukasz J. Krzych, Piotr F. Czempik \\ Department of Anesthesiology and Intensive Care, School of Medicine in Katowice, \\ Medical University of Silesia, Katowice, Poland
}

\begin{abstract}
Background: Fluid resuscitation in massive bleeding may cause coagulation disorders by dilution of platelets and clotting factors or by the impact on their function. The aim of this study was to investigate the effects of balanced crystalloid and colloid solutions on platelets in vitro using complex assessment of coagulation.

Methods: The study group was comprised of 32 American Society of Anesthesiologists physical status class I male volunteers, aged 21-35 (29 \pm 4) years, weighting 59-103 (81.2 \pm 9.8$) \mathrm{kg}$. Whole blood samples were diluted at a 4:1 ratio with the following fluids: balanced crystalloid (Plasmalyte), $6 \%$ hydroxyethyl starch 130/0.4 (Volulyte ${ }^{\circledR}$ ) and succinylated gelatin (Geloplasma ${ }^{\circledR}$ ). Coagulation was assessed using standard morphology, rotational thromboelastometry and aggregometry.

Results: Dilution with all fluids caused statistically significant drop in the number of platelets $(p<0.01)$ but the effect did not differ between solutions $(p>0.05$ for all). Other platelet parameters, such as platelet distribution width, mean platelet volume and platelet-large cell ratio were not affected by the solutions. Hemodilution had no effect on platelet function $(p=0.1)$. Decreased platelet component of clot strength was found for all three fluids ( $p<0.05$ ), although the effect for colloids was more pronounced. Conclusions: The effect of balanced crystalloids and colloids on platelet aggregation was insignificant, even after 20\% volume substitution with the resuscitation fluids. (Cardiol J 2018; 25, 2: 254-259)
\end{abstract}

Key words: platelets, coagulopathy, rotational thromboelastometry, aggregometry, resuscitation fluids

\section{Introduction}

Prudent management of bleeding subjects or those non-bleeding but who are at high risk of hemorrhage is becoming more challenging.

Massive bleeding is considered generally as the loss of one blood volume within a $24 \mathrm{~h}$ period or a $50 \%$ blood volume within $3 \mathrm{~h}$, or a rate of loss of $150 \mathrm{~mL} / \mathrm{min}$. It is a life-threatening condition encountered in many surgical disciplines [1] and in major trauma where it is the primary cause of death in up to one third of patients [2].

According to the current guidelines [3, 4], intravascular volume therapy is the first line treat- ment during massive bleeding. Crystalloids and colloids are essential to restore cardiac output and improve tissue oxygenation in patients with hemorrhagic shock. Simultaneously, both expanders may cause or exaggerate bleeding diathesis. Depending on their constituents and/or carrier solution, they may impair blood coagulation in different ways. The effects may be mediated by dilution of platelets and clotting factors or by the specific influence on their function [5].

The aim herein was to investigate the effects of frequently used balanced crystalloid and colloid solutions on platelets in vitro by means of a complex assessment of coagulation. 


\section{Methods}

The study was approved by the Bioethics Committee of the Medical University of Silesia in Katowice (KNW/0022/KB1/158/15/16). Written informed consent was obtained from 32 American Society of Anesthesiologists (ASA) physical status class I male volunteers, aged $21-35(29 \pm 4)$ years, weighting 59-103 $(81.2 \pm 9.8) \mathrm{kg}$, with no previous history of coagulopathy. No drugs were allowed for 1 week and no alcohol or strenuous physical exercise for 1 day prior to blood sampling.

\section{Sampling}

Venous blood samples were drawn via a $16 \mathrm{G}$ or $18 \mathrm{G}$ indwelling cannula from an antecubital vein of a non-dominant arm. Only minimal stasis was allowed to reduce the risk of pre-analytical error [6]. The first portion of blood $(2 \mathrm{~mL}$ ) was discarded and four blood samples were drawn: baseline (BL, $12.5 \mathrm{~mL}$ ) and one blood sample $(10 \mathrm{~mL})$ for every study fluid. Samples were collected in citrated blood tubes (S-Monovette, Sarstedt, Germany) for complete blood count (CBC) and rotational thromboelastometry analysis, and hirudin blood tubes (S-Monovette, Sarstedt, Germany) for platelet aggregometry. Hirudin test tubes were used because alternative anticoagulants have been shown to affect the results of multiple electrode aggregometry (MEA) [7]. Immediately following collection, samples were processed using rotational thromboelastometry or sent to the central hospital laboratory for analysis. Samples were delivered to the laboratory in person because pneumatic tube transport systems have been shown to affect determinations made with MEA $[8,9]$.

\section{Hemodilution}

The study solutions included balanced crystalloid (Plasmalyte ${ }^{\circledR}$, Baxter) (PL), 6\% hydroxyethyl starch 130/0.4 (Volulyte ${ }^{\circledast}$, Fresenius Kabi) (VL) and succinylated gelatin (Geloplasma ${ }^{\circledR}$, Fresenius Kabi) (GEL). Whole blood samples were diluted with study solutions at a $4: 1$ ratio to make an end-concentration of 20 vol. $\%$ for each solution, equivalent to an infusion of about $1 \mathrm{~L}$ into a $70 \mathrm{~kg}$ person. Taking into account mean weight of volunteers $(81.2 \pm 9.8 \mathrm{~kg})$, the average amount of study solution administered in this recreated scenario was $15 \mathrm{~mL} / \mathrm{kg}$. The investigator performing dilutions was not blinded to study solutions used.

\section{Thromboelastometry}

Rotational thromboelastometry analysis was carried out using a four-channel ROTEM $^{\circledR}$ delta analyzer
(Tem Innovations GmbH, Germany), ROTEM-based assays were allowed to run for $30 \mathrm{~min}$. To minimize a pre-analytical error samples were analyzed immediately after collection. The following whole blood ROTEM assays were performed: INTEM, EXTEM and FIBTEM. The INTEM (describing intrinsic coagulation pathway) uses phospholipid and ellagic acid as activators. The EXTEM is an extrinsic coagulation assay initiated by the addition of a tissue factor. The FIBTEM assay measures effect of fibrin on clot firmness and uses cytochalasin D to inhibit platelets. In both former assays we measured maximum clot firmness $(\mathrm{MCF})$, i.e. parameter influenced by platelets, fibrin and factor XIII. The MCF value was used to calculate maximum clot elasticity (MCE) for both assays using the formula $\mathrm{MCE}=100 \times \mathrm{MCF} /$ /100-MCF. Assessment of platelet contribution to clot strength was measured according to formula $\triangle \mathrm{MCE}=\mathrm{MCE}_{\mathrm{EXTEM}}-\mathrm{MCE}_{\mathrm{FIBTEM}}$.

\section{Aggregometry}

MEA was applied using Multiplate analyzer (Roche Diagnostics GmbH, Germany), along with the thrombin receptor activating peptide-6 (TRAP-6) as a platelet activator. Aggregation units (AU) were calculated. The reference values provided by the laboratory were 941-1563 AU*min.

\section{Standard tests}

Standard laboratory test (SLT) of coagulation included CBC with assessment of platelets (reference value $150000-450000 / \mu \mathrm{L}$ ) and allied parameters, e.g. platelet distribution width (PDW), mean platelet volume (MPV) and platelet-large cell ratio (P-LCR).

\section{Statistical analysis}

The statistical analysis was performed using the licensed MedCalc software (version 16.1 2016, MedCalc Software bvba, Belgium). The quantitative variables are presented as a median (interquartile range $[\mathrm{IQR}])$ or a mean $( \pm$ standard deviation). The type of distribution was verified using Shapiro-Wilk test. The differences between results obtained for particular study solutions were analyzed using repeated measures of ANOVA or Friedman test. Post-hoc comparisons were performed using the Scheffe test or the Conover post-hoc analysis, when appropriate. The level of statistical significance was set at $\mathrm{p}<0.05$.

\section{Results}

Dilution with all study solutions caused a statistically significant drop in the number of platelets 


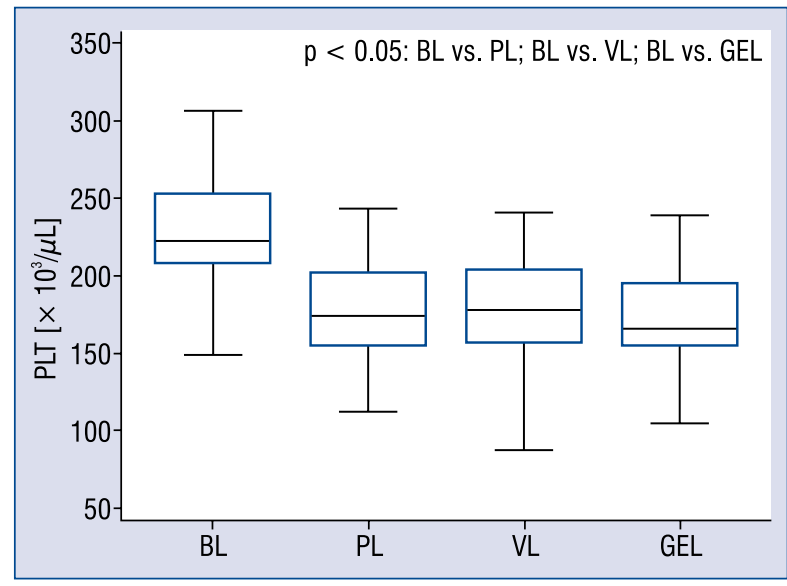

Figure 1. Effect of study solutions on platelet (PLT) count; $\mathrm{BL}$ - baseline; PL - Plasmalyte ${ }^{\circledR} ; \mathrm{VL}-$ Volulyte $^{\circledR}$; GEL - Geloplasma ${ }^{\circledR}$.

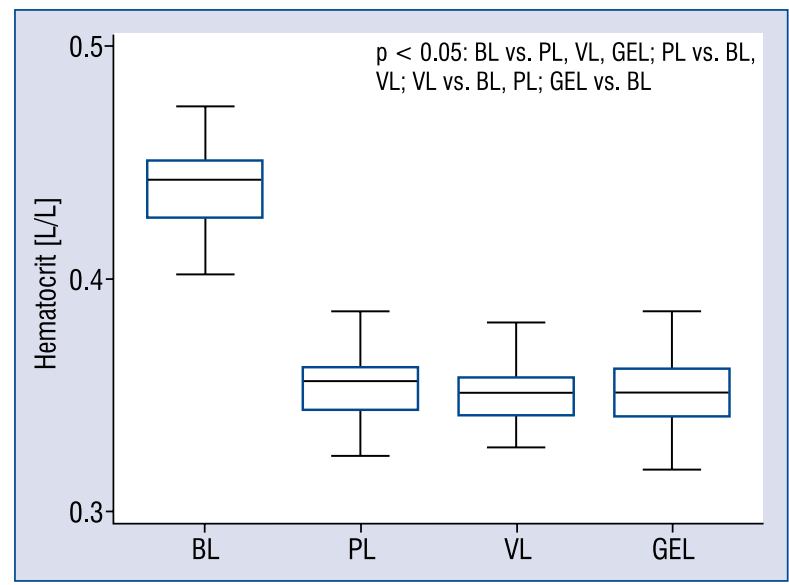

Figure 2. Effect of study solution on hematocrit; $\mathrm{BL}$ - baseline; PL - Plasmalyte ${ }^{\circledR} ; \mathrm{VL}$ - Volulyte $^{\circledR}$; GEL - Geloplasma ${ }^{\circledR}$.

$(\mathrm{p}<0.01)$ but the effect did not differ between solutions ( $p>0.05$ for all) (Fig. 1 ).

The effect of study solution on degree of hemodilution is depicted in Figure 2.

Following administration of PL, VL and GEL blood platelet counts dropped by $21.6 \%, 19.8 \%$ and $25.5 \%$, respectively. Other platelet parameters, such PDW, MPV and P-LCR were not affected by the solutions (Figs. 3-5).

Hemodilution had no effect on platelet function as evidenced by MEA ( $p=0.1$ ) (Fig. 6). The platelet contribution to clot strength, as determined by $\triangle \mathrm{MCE}$, is shown in Figure 7. Decreased platelet component of clot strength was found for all three fluids $(\mathrm{p}<0.05)$, although the effect for colloids was more pronounced. Platelet component of clot strength decreased to $83.2 \%, 78.1 \%, 77.4 \%$ for PL, VL and GEL, respectively.

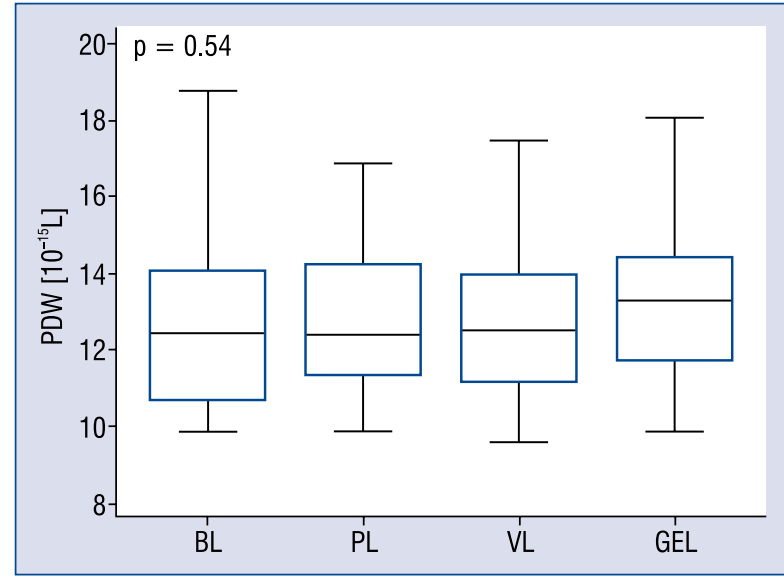

Figure 3. Effect of study solutions on platelet distribution width (PDW); BL — baseline; PL - Plasmalyte ${ }^{\circledR}$; VL - Volulyte ${ }^{\circledR} ;$ GEL - Geloplasma ${ }^{\circledR}$.

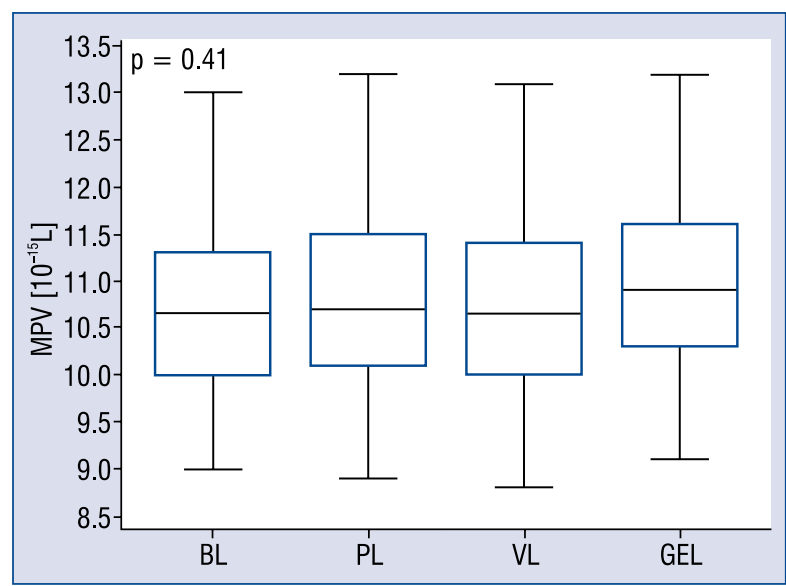

Figure 4. Effect of study solutions on mean platelet volume (MPV); BL_baseline; PL_Plasmalyte ${ }^{\circledR} ; \mathrm{VL}$ - Volulyte ${ }^{\circledR}$; GEL - Geloplasma ${ }^{\circledR}$.

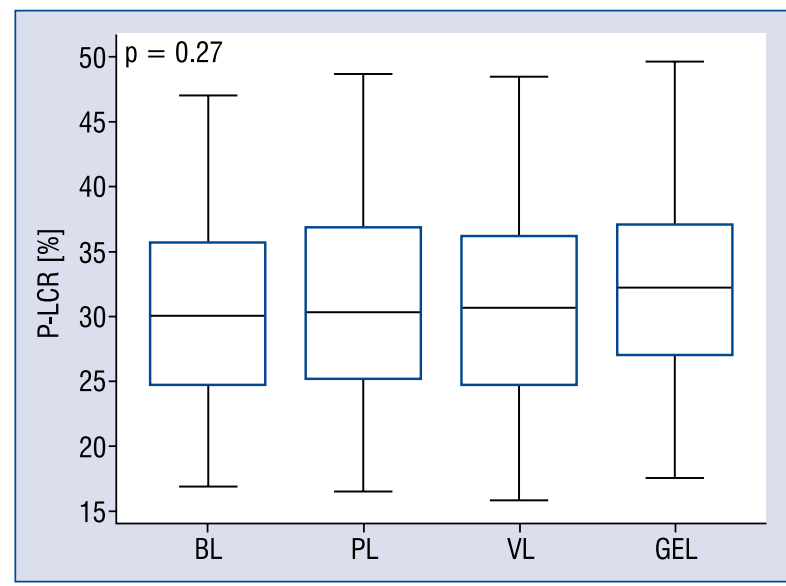

Figure 5. Effect of study solutions on platelet-large cell ratio (P-LCR); BL - baseline; PL - Plasmalyte ${ }^{\circledR}$; VL - Volulyte $^{\circledR} ;$ GEL - Geloplasma $^{\circledR}$. 


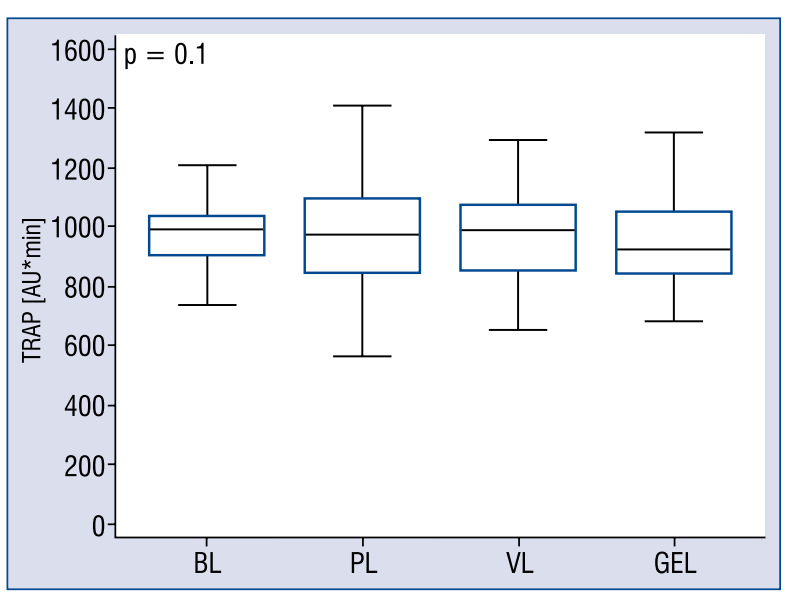

Figure 6. Effect of study solutions on platelet aggregation assessed by thrombin receptor activating peptide (TRAP); $\mathrm{BL}$ - baseline; PL - Plasmalyte ${ }^{\circledR} ; \mathrm{VL}$ - Volulyte $^{\circledR}$; GEL - Geloplasma ${ }^{\circledR}$.

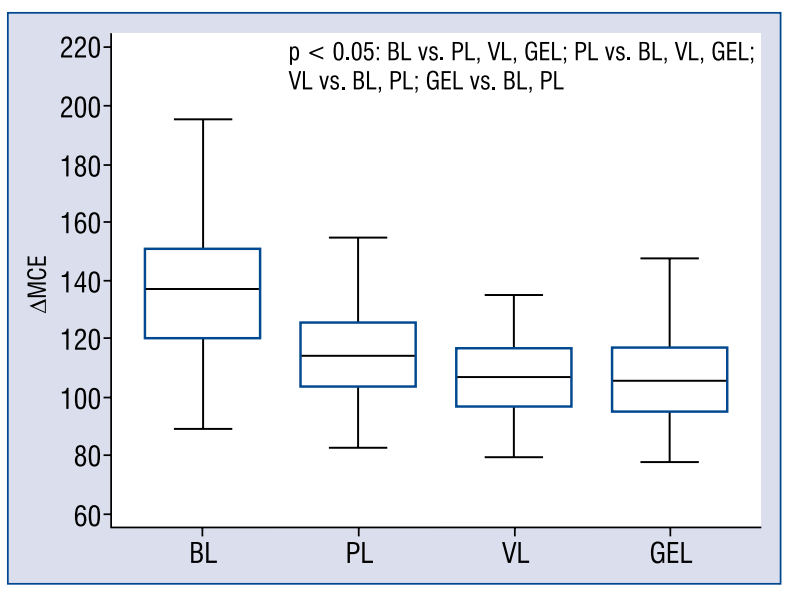

Figure 7. Effect of study solutions on platelet-dependent clot strength; BL - baseline; PL - Plasmalyte $^{\circledR}$; $\mathrm{VL}$ - Volulyte ${ }^{\circledR} ;$ GEL — Geloplasma ${ }^{\circledR}$; MCE — maximum clot elasticity.

\section{Discussion}

Dilution due to aggressive fluid resuscitation leads to decreased concentration of clotting factors and number of platelets. It is interesting as to whether this affect is purely quantitative, or there are some qualitative effect as well. The present study evaluated the effect of three commonly used intravenous fluids on platelets in an in vitro setting. Although 20 vol.\% dilution with study fluids caused significant drop in platelet count, there was no effect on platelet aggregation, and all values for functional and standard tests (with exception of number of platelets) remained within reference laboratory values.

These results are in line with previous investigations showing that crystalloids have less effect on blood coagulation compared to colloids [10]. Contrary to a few other studies, we revealed that platelet function was not affected by the study solutions.

Williams et al. [5] found that platelet function was impaired only at high levels of hemodilution (40\% dilution with 6\% hydroxyethyl starch [HES] $130 / 0.4$ and $0.9 \% \mathrm{NaCl}$ ), as evidenced by light transmission aggregometry (LTA) or MEA. Minor or moderate hemodilution have shown no effect on platelet function. Both fluids examined in this study affected platelet function to a similar, insignificant degree. This discrepancy may be explained by the fact that MEA results were interfered with platelet counts were lower than $150000 / \mu \mathrm{L}$ [11], although large variations have been observed between individuals and normal values of MEA were reported for platelet counts as low as $50000 / \mu \mathrm{L}$ [12].

Treib et al. [13] noticed decreased MPV following HES administration, this effect was seen after several days of its administration. This study did not observe a similar effect, probably due to short exposure to HES.

Liu et al. [14] showed that HES 200/0.5 and $130 / 0.4$ inhibited platelet aggregation ex vivo and the effect was more noticeable with dilutions higher than 20\%, and for HES with higher molecular weight. Shaden et al. [15] showed that only saline-based HES 130/0.42 solutions, but not balanced solution-based HES, administered at a dose of $20 \mathrm{~mL} / \mathrm{kg}$, significantly decreased platelet aggregation in response to adenosine diphosphate (ADP) in healthy volunteers.

In a recent study $\mathrm{Li}$ et al. [16] showed no changes in Multiplate results (ADP-test, TRAPtest) following infusion of up to $1000 \mathrm{~mL}$ of HES $130 / 0.42$ in neurosurgical patients.

Evans et al. [17] investigated the impact of two different gelatin-based fluids on platelet function in vitro. The authors added fluids to make an endconcentration of $40 \%$. Both solutions prevented platelet aggregation induced by ristocetin, although platelet inhibition was more pronounced after Haemaccel $^{\oplus}$, probably due to high $\mathrm{Ca}^{+2}$ content. Haemaccel ${ }^{\oplus}$ also inhibited aggregation induced by other activating factors, namely ADP, platelet activating factor (PAF), collagen, thromboxan A2 (TXA2) and epinephrine. Ristocetin induces platelet aggregation by mechanism involving von Willebrand factor and its glycoprotein Ib receptor. In our study we used TRAP-6 for platelet activa- 
tion. It is known that increasing concentration of $\mathrm{Ca}^{+2}$ impairs platelet aggregation in vitro [18]. In the present study this was not an issue as we used balanced crystalloid and colloids with no extra calcium added.

In a prospective study in which Gelofusine ${ }^{\circledR}$ (4\% succinylated gelatin) was infused into young volunteers, the authors reported a significant increase in bleeding time, impairment of ristocetininduced platelet aggregation, what might have been related to gelatin-induced deficiency of von Willebrand factor [19]. Some authors emphasize a beneficial effect of gelatins as their antiaggregatory properties might provide a degree of protection from thrombosis in trauma patients [20]. Platelets have been observed to be main contributors to increased coagulation after trauma [21]. Similarly HES-induced coagulopathy might counteract the effects of intra-operative activation of coagulation [22].

As far as primary hemostasis is concerned, it is important to distinguish between aggregation and adhesion. None of in vitro tests of coagulation was able to measure impact of vascular endothelium on hemostasis. ROTEM as a diagnostic measure using a whole blood sample, taking into account other physiologic parameters (e.g. temperature, ionized calcium, presence of anticoagulants), is the most accurate test for diagnosing actual coagulopathy. The endothelial component of coagulation is missing in both ROTEM or MEA.

An important topic is the impact of synthetic colloids (used as a priming solution) on bleeding in cardiac surgery performed with cardiopulmonary bypass. Bleeding is common in cardiac surgery. Based on results of the present in vitro research, the use of succinylated gelatin and HES as a priming solution should be cautious [23].

\section{Limitations of the study}

First of all, only 20 vol.\% blood dilution was made, what may be insufficient to observe coagulation abnormalities. Clinically relevant effects of synthetic plasma expanders on bleeding are present when large volumes $(>20 \mathrm{~mL} / \mathrm{kg}$ ) are infused or when patients present a priori with coagulopathy [20]. This study attempted to mitigate this drawback using sensitive functional testing, i.e. $\triangle \mathrm{MCE}$ in ROTEM and MEA analysis. The study herein ran two assays simultaneously, one with platelet inhibitor and one without, to identify specific coagulopathy caused by platelet defect.
The effect of the study solutions on platelet component of clot strength as measured by ROTEM parameters showed decreased MCE for all three study solutions, which was more pronounced for HES and gelatin. Measuring platelet-dependent clot strength by elasticity, as opposed to clot amplitude, is sound because the relationship between clot amplitude and clot elasticity is nonlinear and clot elasticity, unlike clot amplitude, reflects the force of the blood clot resisting rotation within the device [24]. Moreover clot strength, as measured by clot amplitude, depends not only on platelets, but also on fibrinogen and factor XIII concentrations, although the relative contribution of platelets is approximately $80 \%$ [21]. Based on clot elasticity, platelet contribution is $87-94 \%$ [25]. Secondly, the quantity of von Willebrand factor may impact clot stability and resulting bleeding time [19], postoperative blood loss [26], however in the present study its concentration was not measured. Thirdly, MEA was used for testing platelet function, whereas the most objective test of platelet function is LTA. Another limitation is the fact that observations are based on in vitro experiments what may differ from conditions caused by true fluid resuscitation, including recruitment of additional resources of the coagulation system, response to tissue and endothelial injuries, as well as changes in kinetics, volume of distribution or acid-base balance. Therefore in vivo studies deliver more physiological data. On the other hand, findings in healthy volunteers would also differ from those in actual massive bleeding. Finally, there was no control group for balanced solutions. It was however revealed that carrier solution had little impact on platelet aggregation and clot formation [15, 27].

\section{Conclusions}

The effect of balanced crystalloids and colloids on platelet aggregation is insignificant, even after $20 \%$ volume substitution.

\section{Acknowledgements}

We express our gratitude to the personnel of the central hospital laboratory in the Silesian Centre for Heart Diseases in Zabrze for providing determinations for this study.

Conflict of interest: Ł.J. Krzych, P.F. Czempik - study supported by a grant sponsored by the Adamed corporation. 


\section{References}

1. Starczewska MH, Giercuszkiewicz D, Niewiński G, et al. Perioperative bleeding in patients undergoing liver transplantation. Anaesthesiol Intensive Ther. 2016; 48(1): 34-40, doi: 10.5603/ AIT.a2015.0020, indexed in Pubmed: 25830935.

2. Tieu BH, Holcomb JB, Schreiber MA. Coagulopathy: its pathophysiology and treatment in the injured patient. World J Surg. 2007; 31(5): 1055-1064, doi: 10.1007/s00268-006-0653-9, indexed in Pubmed: 17426904.

3. Kozek-Langenecker SA, Ahmed AB, Afshari A, et al. Management of severe perioperative bleeding: guidelines from the European Society of Anaesthesiology. Eur J Anaesthesiol. 2013; 30(6): 270-382, doi: 10.1097/EJA.0b013e32835f4d5b, indexed in Pubmed: 23656742.

4. Rossaint R, Bouillon B, Cerny V, et al. The European guideline on management of major bleeding and coagulopathy following trauma: fourth edition. Crit Care. 2016; 20: 100, doi: 10.1186/ s13054-016-1265-x, indexed in Pubmed: 27072503.

5. Williams P, Yang K, Kershaw G, et al. The effects of haemodilution with hydroxyethyl starch 130/0.4 solution on coagulation as assessed by thromboelastography and platelet receptor function studies in vitro. Anaesth Intensive Care. 2015; 43(6): 734-739, indexed in Pubmed: 26603798.

6. Lima-Oliveira G, Lippi G, Salvagno GL, et al. Venous stasis and whole blood platelet aggregometry: a question of data reliability and patient safety. Blood Coagul Fibrinolysis. 2015; 26(6): 665668, doi: 10.1097/MBC.0000000000000342, indexed in Pubmed: 26154610 .

7. Karlsson M, Roman-Emanuel C, Thimour-Bergström L, et al. Sampling conditions influence multiple electrode platelet aggregometry in cardiac surgery patients. Scand Cardiovasc J. 2013; 47(2): 98-103, doi: 10.3109/14017431.2012.743672, indexed in Pubmed: 23098207.

8. Thalén S, Forsling I, Eintrei J, et al. Pneumatic tube transport affects platelet function measured by multiplate electrode aggregometry. Thromb Res. 2013; 132(1): 77-80, doi: 10.1016/j. thromres.2013.04.020, indexed in Pubmed: 23706534.

9. Bolliger D, Seeberger MD, Tanaka KA, et al. Pre-analytical effects of pneumatic tube transport on impedance platelet aggregometry. Platelets. 2009; 20(7): 458-465, doi: 10.3109/09537100903236462, indexed in Pubmed: 19852683.

10. Skhirtladze K, Base EM, Lassnigg A, et al. Comparison of the effects of albumin $5 \%$, hydroxyethyl starch $130 / 0.46 \%$, and Ringer's lactate on blood loss and coagulation after cardiac surgery. Br J Anaesth. 2014; 112(2): 255-264, doi: 10.1093/bja/aet348, indexed in Pubmed: 24169821.

11. Stissing T, Dridi NP, Ostrowski SR, et al. The influence of low platelet count on whole blood aggregometry assessed by Multiplate. Clin Appl Thromb Hemost. 2011; 17(6): E211-E217, doi: 10.1177/1076029610397183, indexed in Pubmed: 21406416.

12. Hanke AA, Roberg K, Monaca E, et al. Impact of platelet count on results obtained from multiple electrode platelet aggregometry (Multiplate). Eur J Med Res. 2010; 15(5): 214-219, indexed in Pubmed: 20562061.

13. Treib J, Haass A, Pindur G, et al. [Decrease of thrombocyte volume after several days infusion of highly substituted medium molecular weight hydroxyethyl starch (HAES 200/0.62)]. Wien Klin Wochenschr. 1996; 108(1): 20-23, indexed in Pubmed: 8677658.

14. Liu FC, Liao CH, Chang YW, et al. Hydroxyethyl starch interferes with human blood ex vivo coagulation, platelet func- tion and sedimentation. Acta Anaesthesiol Taiwan. 2009; 47(2): 71-78, doi: 10.1016/S1875-4597(09)60027-8, indexed in Pubmed: 19527967.

15. Schaden E, Wetzel L, Kozek-Langenecker S, et al. Effect of the carrier solution for hydroxyethyl starch on platelet aggregation and clot formation. Br J Anaesth. 2012; 109(4): 572-577, doi: 10.1093/bja/aes229, indexed in Pubmed: 22791802.

16. Li N, Statkevicius S, Asgeirsson B, et al. Effects of different colloid infusions on ROTEM and Multiplate during elective brain tumour neurosurgery. Perioper Med (Lond). 2015; 4: 9, doi: 10.1186/s13741-015-0019-7, indexed in Pubmed: 26425342.

17. Evans PA, Glenn JR, Heptinstall S, et al. Effects of gelatin-based resuscitation fluids on platelet aggregation. Br J Anaesth. 1998; 81(2): 198-202, doi: 10.1093/bja/81.2.198, indexed in Pubmed: 9813523.

18. Heptinstall S. The use of a chelating ion-exchange resin to evaluate the effects of the extracellular calcium concentration on adenosine diphosphate induced aggregation of human blood platelets. Thromb Haemost. 1976; 36(1): 208-220, indexed in Pubmed: 1036811.

19. de Jonge E, Levi M, Berends F, et al. Impaired haemostasis by intravenous administration of a gelatin-based plasma expander in human subjects. Thromb Haemost. 1998; 79(2): 286-290, indexed in Pubmed: 9493577.

20. Levi M, Jonge Ede. Clinical relevance of the effects of plasma expanders on coagulation. Semin Thromb Hemost. 2007; 33(8): 810-815, doi: 10.1055/s-2007-1000370, indexed in Pubmed: 18175286.

21. Harr JN, Moore EE, Chin TL, et al. Platelets are dominant contributors to hypercoagulability after injury. J Trauma Acute Care Surg. 2013; 74(3): 756-62; discussion 762, doi: 10.1097/ TA.0b013e3182826d7e, indexed in Pubmed: 23425732.

22. Nilsson CU, Strandberg K, Engström M, et al. Coagulation during elective neurosurgery with hydroxyethyl starch fluid therapy: an observational study with thromboelastometry, fibrinogen and factor XIII. Perioper Med (Lond). 2016; 5: 20, doi: 10.1186/ s13741-016-0046-z, indexed in Pubmed: 27540479.

23. Krzych ŁJ, Czempik PF. Hooves better than potatoes: in vitro effects of balanced crystalloid and colloids on functional parameters of coagulation and fibrinolysis. Pol Arch Intern Med. 2017; 127(3): 209-211, doi: 10.20452/pamw.3981, indexed in Pubmed: 28322215.

24. Solomon C, Ranucci M, Hochleitner G, et al. Assessing the Methodology for Calculating Platelet Contribution to Clot Strength (Platelet Component) in Thromboelastometry and Thrombelastography. Anesth Analg. 2015; 121(4): 868-878, doi: 10.1213/ ANE.0000000000000859, indexed in Pubmed: 26378699.

25. Nielsen VG, Geary BT, Baird MS. Evaluation of the contribution of platelets to clot strength by thromboelastography in rabbits: the role of tissue factor and cytochalasin D. Anesth Analg. 2000; 91(1): 35-39, indexed in Pubmed: 10866883.

26. Mazur P, Plicner D, Zdziarska J, et al. Decreased von Willebrand factor ristocetin cofactor activity and increased ADAMTS13 antigen increase postoperative drainage after coronary artery bypass grafting. Eur J Cardiothorac Surg. 2014; 45(2): e26-e32, doi: 10.1093/ejcts/ezt527, indexed in Pubmed: 24351199.

27. Casutt M, Kristoffy A, Schuepfer G, et al. Effects on coagulation of balanced (130/0.42) and non-balanced (130/0.4) hydroxyethyl starch or gelatin compared with balanced Ringer's solution: an in vitro study using two different viscoelastic coagulation tests ROTEMTM and SONOCLOTTM. Br J Anaesth. 2010; 105(3): 273-281, doi: 10.1093/bja/aeq173, indexed in Pubmed: 20659913. 